\title{
Dynamics of Homogeneous Moment Neuronal Networks with Context Units
}

\author{
Yonglin Wang $^{1}$ and Xuyan Xiang ${ }^{2 *}$ \\ ${ }^{1}$ College of Computer Science, Hunan University of Arts and Science, Changde 415000, China; ${ }^{2}$ College of Mathematics \\ and Computational Science, Hunan University of Arts and Science, Changde 415000, China
}

\begin{abstract}
After discussions of a few relevant patents of neural networks, a model description of moment neuronal networks with context units is given by introducing intra-layer inputs. The dynamics of homogeneous networks derived from the intra-inputs are explored, including the input-output relationships and the stability of such network. It is shown how the spontaneous activity is propagated across the homogeneous feed-forward networks with context units. Due to a more biologically reasonable context unit, such network offers a significant advantage over the recent moment neuronal networks in that it can enhance or weaken the dynamics of network by the adjustment of the parameters from context unit, based on those from network itself, and it can lead to some unexpectedly dynamic properties. In this paper we highlight the key and sophisticated role played by the context unit in dynamics of such network.
\end{abstract}

Keywords: Context unit, dynamics, intra- and inter-layer interactions, moment neuronal networks, stability.

\section{INTRODUCTION}

In recent years, there has been a significant growth in the field of biologically-inspired computation, and current computational neural models owe more to their biological counterparts than their predecessors. For instance, the integrate-and-fire (IF) model [1-3], incorporates more of the dynamics of biological neurons than the classical approach to neural modeling. Patent 8,346,692, titled "Spatio-temporal pattern recognition using a spiking neural network and processing thereof on a portable and/or distributed computer" [4], provides a spiking neural network with a layer of connected spiking neurons exchanging signals, in which a pattern is submitted to the spiking neural network for generating sequences of spikes, which are modulated over time by the spiking synchronization. Patent 8,515,885, titled "Neuromorphic and synaptronic spiking neural network with synaptic weights learned using simulation" [5], provides neuromorphicsynaptronic systems, implementing spiking neural network with synaptic weights.

Many of the engineering applications which have applied biophysical models, have used the IF model as the main computational unit. Patent 6,262,678, titled "Current-mode spike-based analog-to-digital conversion" [6], invents A/D conversion of a current input with integrate-and-fire spiking neurons that the speed of the conversion is augmented in a pipelined topology. Patent 7,430,546, titled "Applications of an algorithm that mimics cortical processing" [7], invents an information processing system having neuron-like signal processors that are interconnected by synapse-like processing junctions that simulates and extends capabilities of biological neural networks. The information processing systems uses IF neurons and Temporally Asymmetric Hebbian learning (spike timing-dependent learning) to adapt the synaptic strengths. This neural network is well-suited for hardware implementations. Such hardware implementation may be used for predicting and recognizing audiovisual information or for improving cortical processing by a prosthetic device.

It has emerged that the spike activity of a neuron is decided not only by the mean input activity (mean firing rate), but also by higher order statistics of its inputs, including fluctuations and correlations [1, 8-14]. A progress in this direction, was made by [15] with their theory of moment neuronal networks (MNN). This takes into account both the first and the second order statistics of spike trains, generalizing the case of Poisson synaptic inputs to more biologically plausible renewal processes. Therefore, the MNN framework presented in [15] can be considered as an attempt toward a general framework of computation with stochastic systems. In this respect, it would play the role of the central limit theorem in the probability theory, whereas earlier approaches based exclusively upon the mean could be likened to the law of large numbers.

Further, by introducing intra- layer inputs, Xiang et al. [16] developed a more biologically reasonable MNN with intra- and inter-layer interactions, which is analogous to the "networks with context units" in ANNs (e. g. [17], Patent WO/2013/169805, titled "Spiking neural network feedback apparatus and methods" [18], discloses the apparatus and methods for feedback in a spiking neural network. In which, spiking neurons receive sensory stimulus and context signal that correspond to the same context.), and applied a back- 
propagation learning rule to train the networks. It shows that even a single unit in such system, as analogous to [19-23], is able to perform various complex non-linear tasks like the XOR problem, and that it can train the output variance by directly introducing the variance term in the error presentation rather than indirectly adding a weight penalty term as in ANNs (e.g. [24, 25], JP Patent 2,000,200,256, titled "Learning method for recursive neutral network and recording medium where learning program for recursive neural network is recorded" [26], provides a learning method that a term of a proper scale of internal state representation is provided for the evaluation function to be evaluated in addition to terms including an error term and a penalty term and the maximum likelihood estimation is performed for the coefficients of the respective terms.). Moreover, one can reach the trade-off between the output bias and variance by optimizing the penalty factor due to a specific learning task.

In this paper we will explore how the spontaneous activity is propagated across the homogeneous feed forward network with the varying parameters, especially from the context units, i.e. the intra- layers inputs. Here we show the different properties on both input-output firing relationship and stability of network from MNN. Due to a more biologically reasonable context units (i.e. intra- layer inputs), such network offers a significant advantage over the recent MNN in that it can change the dynamics of network by the adjustment of the parameters from context unit (and from network itself), and it can lead to some unexpectedly dynamic properties. The context unit plays a very sophisticated role in the dynamics of such network. It can enhance or weaken the firing outputs by varying on the ratio $r^{i a}$ or the size $p^{i a}$ of context unit, based on the ratio $r$ and the size $\mathrm{p}$ of network itself, i.e. inter layer.

This paper is organized as follows. The framework for MNN with context units is described in Section 2. The dynamics of homogeneous network varied from context units are explored in Section 3.

\section{MNN WITH CONTEXT UNITS}

To derive the dynamics, we first introduce the framework of MNN with context units, i.e. with intra- and interinteractions. We begin by considering the single IF neuron $[3,27]$ as follows. Suppose the $i$-th neuron in the $(k+1)$ th layer receives excitatory postsynaptic potentials (EPSPs) and inhibitory postsynaptic potentials (IPSPs). When the membrane potential $V_{i}^{(k+1)}(t)$ of it is between its resting state $V_{\mathrm{r}}$ and its threshold $V_{\mathrm{t}}$, it satisfies the following dynamics:

$d V_{i}^{(k+1)}(t)=-L\left(V_{i}^{(k+1)}(t)-V_{r}\right) d t+d I_{i, s y n}^{(k+1)}(t)$

Where, $L$ is the decay rate, $I_{i, s y n}^{(k+1)}(t)$ the synaptic input given by

$$
\begin{aligned}
& d I_{i, s y n}^{(k+1)}(t)=\sum_{j=1}^{p^{(k, \text {,ir }}} \omega_{i j}^{E,(k), \mathrm{ir}} d N_{j}^{E,(k), \mathrm{ir}}(t)-\sum_{j=1}^{q^{(k), \mathrm{ir}}} \omega_{i j}^{I,(k), \mathrm{ir}} d N_{j}^{I,(k), \mathrm{ir}}(t) \\
& +\sum_{j=1}^{p^{(k+1), \mathrm{ia}}} \omega_{i j}^{E,(k+1), \mathrm{ia}} d N_{j}^{E,(k+1), \mathrm{ia}}(t)-\sum_{j=1}^{q^{(k+1), \mathrm{ia}}} \omega_{i j}^{I,(k+1), \mathrm{ia}} d N_{j}^{I,(k+1), \mathrm{ia}}(t)
\end{aligned}
$$

Here $\omega_{i j}^{E,(k)}$ is the magnitude of EPSPs, $\omega_{i j}^{E,(k), \text { ir }}$ is the magnitude of IPSPs, $\left\{N_{i}^{E,(k), \text { ir }}(t)\right\}$ and $\left\{N_{i}^{\mathrm{l},(k), \text { ir }}(t)\right\}$ are renewal process arriving from the $i$-th and $j$-th synapse, $p^{(k)}$ and $q^{(k)}$ are the total number of active excitatory and inhibitory synapses in the $k$-th layer. All notations with the superscript ia implies that they come from intra-layer interactions, similar to the 'networks with context unit' [17]. It would present a more biologically reasonable networks architecture than the moment neuronal networks. When $V_{i}^{(k+1)}(t)$ crosses the membrane threshold $V_{t}$ from below, a spike is generated and the membrane resets to its resting potential $V_{r}$.

$$
\text { Let }\left\langle T_{i}^{\mathrm{E},(k), \mathrm{ix}}\right\rangle \text { and } \operatorname{Var}\left\langle T_{i}^{\mathrm{E},(k), \mathrm{x}}\right\rangle \text { as the mean and variance of }
$$

the interspike intervals (ISIs) of the renewal process $\left\{\mathrm{N}_{i}^{E,(k)}(t)\right\}$ respectively. To model the synaptic input similar to how it appears in biological neurons, for the $i$-th neuron in the $k$-th layer, we choose a more biological plausible socalled usual approximation scheme [15] to approximate the renewal process $\left\{N_{i}^{E, k}(t)\right\}$

$d N_{i}^{E, k, i x}(t) \sim \mu_{i}^{E,(\mathrm{k}), \mathrm{ix}} d t+\sigma_{i}^{E,(\mathrm{k}), \mathrm{ix}} d B_{i}^{E,(\mathrm{k}), \mathrm{ix}}(t)$

With,

$$
\mu_{i j}^{\mathrm{E},(k), \mathrm{ix}}=\frac{1}{\left\langle T_{i j}^{E,(k), i x}\right\rangle+T_{r e f}}, \quad\left(\sigma_{i j}^{\mathrm{E},(k), \mathrm{ix}}\right)^{2}=\frac{\operatorname{Var}\left(T_{i}^{\mathrm{E},(k), \mathrm{ix}}\right)}{\left\langle T_{i j}^{\mathrm{E},(k), \mathrm{ix}}\right\rangle^{3}}
$$

Where, the superscript $i x$ could be either ir or $i a$ as stated before, and $T_{r e f}$ is the refractory period.

For the concise of notation, we take the intra-input from the $(k+1)$ th layer as external context input paralleled to the $k$ th layer, which is denoted by the superscript $i a$. Furthermore, we suppose that $q^{(k) \text {,ix }}=p^{(k), \mathrm{ix}}, \mu^{(k) \text {,ix }} \equiv$ $\mu^{\mathrm{I},(k), \mathrm{ix}}=\mu^{\mathrm{E},(k), \mathrm{ix}}, \sigma^{(k), \mathrm{ix}} \equiv \sigma^{\mathrm{I},(k), \mathrm{ix}}=\sigma^{\mathrm{E},(k), \mathrm{ix}}, \quad$ and $\omega_{i j}^{\mathrm{L},(k), \mathrm{ir}}=\gamma^{i r} \omega_{i j}^{\mathrm{E},(k), \mathrm{ir}} \equiv \gamma \omega_{i j}^{(k), \mathrm{ir}} \equiv \gamma \omega_{i j}^{(k)}, \omega_{i j}^{\mathrm{I},(k), \mathrm{ia}}=\gamma^{i a} \omega_{i j}^{\mathrm{E},(k), \mathrm{ia}} \equiv \gamma^{i a} \omega_{i j}^{(k), \mathrm{ia}}$, $\rho_{m n}^{(k)} \equiv \rho_{m n}^{(k), i}$, then the equation (2) can be approximated as (see [16])

$d I_{i, s y n}^{(k+1)}(t) \equiv \bar{\mu}_{i}^{(k)} d t+\bar{\sigma}_{i}^{(k)} d B(t)$

Where,

$$
\begin{aligned}
& \bar{\mu}_{i}^{(k)}=\sum_{j=1}^{p^{(k)}} \omega_{i j}^{(k)} \mu_{j}^{(k)}(1-r)+\sum_{j=1}^{p^{(k) \text {,ia }}} \omega_{i j}^{(k) \text {,ia }} \mu_{j}^{(k) \text {,ia }}\left(1-r^{i a}\right) \\
& \left(\sigma_{i}^{(k)}\right)^{2}=\sum_{m, n=1}^{p^{(k)}} \omega_{i m}^{(k)} \sigma_{m}^{(k)} \omega_{i n}^{(k)} \sigma_{n}^{(k)} \rho_{m n}^{(k)}\left(1+r^{2}\right) \\
& +\sum_{m, n=1}^{p^{(k) \text {,ia }}} \omega_{i m}^{(k), \text { ia }} \sigma_{m}^{(k), \text { ia }} \omega_{i n}^{(k), \text { ia }} \sigma_{n}^{(k), \text {,a }} \rho_{m n}^{(k), \text { ia }}\left(1+\left(r^{i a}\right)^{2}\right)
\end{aligned}
$$

Here $r^{i a}$ is the ratio between inhibitory inputs and excitatory inputs from the intra-layer; $\rho_{j s}^{(k), \text { ir }}$ and $\rho_{j s}^{(k), \text { ia }}$ are correlation coefficient between the $j$-th and $s$-th input from inter- and intra- layer. It is worth noting here, that the model's 
description of synaptic input presents it in terms of input mean and variance.

Notice that, according to the anatomy of the nervous system, we can suppose that $q^{(k) \text {,ir }}=0$, which is equivalent to $r \equiv r^{i r} \equiv 0$ in equation (5).

In terms of Siegert's expression [28], we have the expression of all moments of the output ISIs. For the mean and variance, we have

$$
\begin{gathered}
\left\langle T_{i}^{(k+1)}\right\rangle=\frac{2}{L} \int_{A_{i}^{(k)}\left(V_{r}\right)}^{A_{i}^{(k)}\left(V_{t}\right)} a(x) d x, \\
\operatorname{Var}\left(T_{i}^{(k+1)}\right)=\frac{4}{L^{2}} \int_{A_{i}^{A_{i}}\left(A_{(}\right)\left(V_{r}\right)}^{(k)} h(x) d x,
\end{gathered}
$$

Where,

$$
\begin{aligned}
& A_{i}^{(k)}(y)=\frac{y L-\bar{\mu}_{i}^{(k)}}{\bar{\sigma}_{i}^{(k)} \sqrt{L}}, \\
& a(x)=\exp \left(x^{2}\right) \int_{-\infty}^{x} \exp \left(-u^{2}\right) d u, \\
& h(x)=\exp \left(x^{2}\right) \int_{-\infty}^{x} \exp \left(-u^{2}\right) a^{2}(u) d u
\end{aligned}
$$

To complete the description of the moment mapping we need to consider the relationship between the input and the output correlations. Unfortunately, such a relationship is difficult to derive analytically, so we had to resort to numerical simulations. The numerical results with no intrainputs in [15] indicate that the input-output relationship is close to the identity, independently of $r$ and $\mu$.

Hence, we can assume that the following heuristic relationship holds:

$$
\rho_{i j}^{(k+1)}=\frac{\sum_{m, n} \omega_{i m}^{(k)} \sigma_{m}^{(k)} \omega_{j n}^{(k)} \sigma_{n}^{(k)} \rho_{m n}^{(k)}}{\sqrt{\sum_{m, n} \omega_{i m}^{(k)} \sigma_{m}^{(k)} \omega_{i n}^{(k)} \sigma_{n}^{(k)} \rho_{m n}^{(k)}} \sum_{m, n} \omega_{j m}^{(k)} \sigma_{m}^{(k)} \omega_{j n}^{(k)} \sigma_{n}^{(k)} \rho_{m n}^{(k)}}
$$

Note that the right-hand side of Eq. (9) is the correlation of the inputs to the $i$ th and the $j$ th neurons in the $(k+1)$ th layer, which includes the intra- and inter- input correlations.

According to the discussion in the previous sections, the mean and variance of the output in $(k+1)$-th layer can be given as follows:

$$
\begin{aligned}
& \mu_{i}^{(k+1)}=\frac{1}{\left(\left\langle T_{i}^{(k+1)}\right\rangle+T_{r e f}\right)} \equiv f_{i}^{(k+1)} \equiv f_{i}^{(k+1)}\left(\mu_{j}^{(k)}, \sigma_{j}^{(k)}\right) \\
& \left.\left(\sigma_{i}^{(k+1)}\right)^{2}=\operatorname{Var}\left(T_{i}^{(k+1)}\right) T_{i}^{(k+1)}\right\rangle^{-3} \equiv g_{i}^{(k+1)} \equiv g_{i}^{(k+1)}\left(\mu_{j}^{(n)}, \sigma_{j}^{(k)}\right)
\end{aligned}
$$

The above relationship between inputs and outputs lays the foundation of the moment neural networks (MNNs) with intra- and inter- interactions.

The topology used in the definition of MNN with intraand inter- interactions is mainly based on the classic feedforward MLP network architecture, accompanied with a context input units for each layer.

\section{DYNAMICS OF NETWORKS WITH SIMULATIONS}

The question we intend to address here is how a spontaneous activity can be maintained in a feed-forward network. For all simulations, the values of the decay rate, threshold and resting potential were set equal to $L=1 / 20$ $\mathrm{ms}^{-1}, V_{\mathrm{t}}=20 \mathrm{mV}$, and $V_{r}=0 \mathrm{mV}$ respectively. The same parameters have been employed elsewhere [27], and are thought to be in the physiological range for visual cortex cells and in agreement with most published results [12, 29]. All simulations were carried out with MATLAB.

We focus on a homogeneous network, where all weights, afferent means, and variances were set to be identical. Now the quantities in (5) reduce to

$$
\begin{aligned}
& \mu_{1}^{(k)}=\mu_{j}^{(k)}, \sigma_{1}^{(k)}=\sigma_{j}^{(k)}, \rho_{12}^{(k)}=\rho_{m n}^{(k)}=\rho, m \neq n, \\
& \mu_{1}^{(k) \text {,ia }}=\mu_{j}^{(k) \text {,ia }}, \sigma_{1}^{(k) \text {,ia }}=\sigma_{j}^{(k) \text {,ia }}, \rho_{12}^{(k) \text {,ia }}=\rho_{m n}^{(k) \text {,ia }}=\rho^{\text {ia }}, m \neq n .
\end{aligned}
$$

As we discussed before, the propagation of correlation becomes trivial in such case since all cells become fully correlated after the first layer. To avoid this, we clamped the correlation coefficient, i.e., we set

$$
\rho_{m n}^{(k)}=\rho, \rho_{m n}^{(k), \text { ia }}=\rho^{i a}, m \neq n .
$$

In simulations we set $\rho^{i a}=\rho=0$ or 0.1 , in agreement with experimental data reported in the literature $[9,14,15]$; and we assume that the context units from each intra-layer, including numbers of neurons, afferent means and variances are identical, i.e.

$$
\mu_{1}^{(1)}=\mu_{1}^{(k)}, \rho^{(1), i a}=\rho^{(k), \text { ia }}=\rho^{i a} .
$$

Without intra-inputs, [15] (Fig. 5) derived the propagation of activity in a homogeneous feed-forward MNN with $p=100, \quad \rho_{i j}=0, \omega_{i j}=\omega=0.5$ and $r \in(0,0.4)$ for $\mu_{1}^{(1)}=100 \mathrm{~Hz}$ and $50 \mathrm{~Hz}$, and showed that after the first few layers, neurons are found to be either silent (e.g. $\mathrm{r}=0.4$ ) or firing at relatively high frequency (e. g. $\mathrm{r}=0.1$ ) about 100 $\mathrm{Hz}$ for different initial CV. As an analogous to [15], we stop our simulation when the firing rate is slower than $0.001 \mathrm{~Hz}$. The coefficient of variation [16], here, quantifies the irregularity of a spike train. If $C V=0$, the spike train is regular, otherwise the spike train is random. Thus we choose $r=0.1$ and $r=0.5$ to illustrate how the activity is propagated across the networks, varied from $r^{i a}$ and $p^{i a}$, on assumption that $C_{1}^{(\mathrm{k})}=C_{1}^{(k) \text {,ia }}=1$ (A larger $r$ leads to be certainly silent due to a stronger inhibition). Unless otherwise specified, the initial $C V$ in all simulations is always 1 , and the weight is 0.5 .

In Fig. (1), we show the results obtained for various values of $\mu^{(1)}$ and $\sigma^{(1)}$ (we reported the coefficient of variation $C^{(\mathrm{k})}=\sigma^{(\mathrm{k})} / \mu^{(\mathrm{k})}$. As stated in [15], each data point $\left(\mu^{(\mathrm{k})}, C^{(\mathrm{k})}\right)$ is connected with $\left(\mu^{(\mathrm{k}+1)}, C^{(\mathrm{k}+1)}\right)$ to illustrate how the activity is propagated across the networks. For 

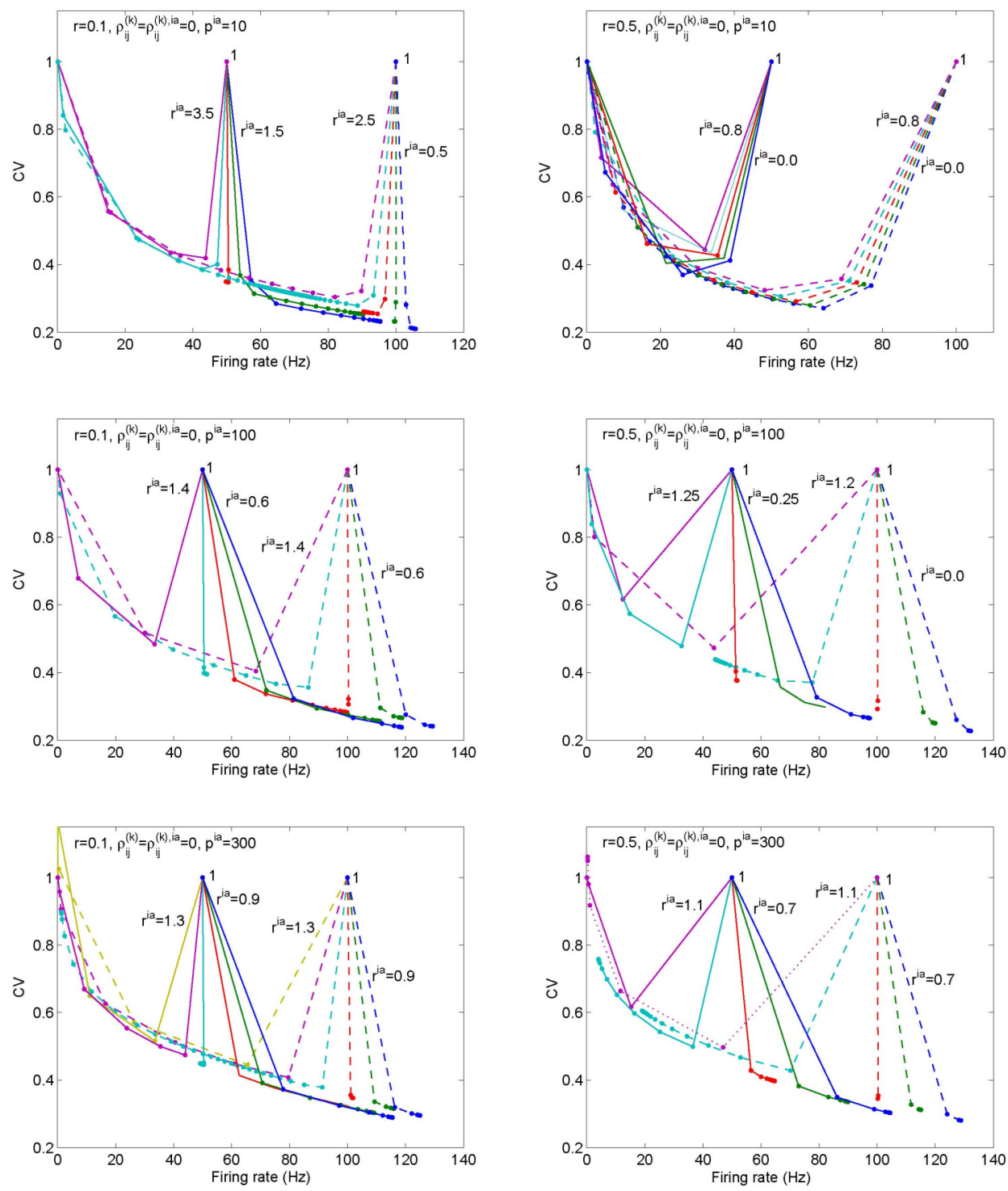

Fig. (1). Propagation of activity in a homogeneous feedforward MNN with context units. For each layer $k$, the output firing rate $\left(\mu_{1}^{(\mathrm{k})}\right)$ and the coefficient of variation $(C V)$ of the interspike intervals $\left(C_{1}^{(\mathrm{k})}\right)$ are reported on the abscissa and the ordinate. Points labeled 1 correspond to the first layer; points corresponding to successive layers are connected by lines. Results were obtained for $p^{(\mathrm{k})}=p=100, p_{i j}=p_{i j}^{i a}=0$, $\mu_{1}^{(1)}=50 \mathrm{~Hz}$ (solid lines), $100 \mathrm{~Hz}$ (dashed lines), and $r=0.1$ (left), $r=0.5$ (right), and $p^{i a}=10$ (top), 100 (middle) and 300 (bottom). In each panel, the ratios from intra-layer $\left(r^{i a}\right)$ are evenly spaced.

$r=0.1$ (left), after the first few layers, neurons are found to be either silent or firing at relatively high frequency for all $r^{i a}$ and $p^{i a}$. For $r=0.5$ (right), however, the situation has changed.

First, top right shows even if there is no inhibitory input $\left(r^{i a}=0\right)$, to be silent is certainty on fewer intra- input neurons. Second, middle right and bottom right show that not all case are to be silent, for examples, $r^{a}=0.9$ with $\mu_{1}^{(1)}=100$ (middle right) and $r^{i a}=1$ with $\mu_{1}^{(1)}=50$ (bottom right), are exclusively found to be firing at relatively low frequency.

In Fig. (2), we further show how the activity is propagated across the networks with the varying numbers of intra-layer neurons $p^{i a}$ (left) and the ratio of intra- inputs $r^{i a}$ (right), respectively. As stated earlier, for $r=0.5$ with fewer intra- input neurons $\left(p^{i a}=10\right)$, the network is certainty to be silent (see Fig. (1), top right). However with increasing size of intra- input units, the network might be fixing or firing at relatively high frequency (see Fig. 2, left). Right panel of Fig. (2) also shows that not all networks are certainty to be silent. Instead, they fire at low or relatively high frequency. It actually shows the networks can be stable at low frequency, see discussions later.

Then we show how the relationship between input and output firing rate is varied from the context units or intralayer input units. Figs. (3 and 4) show how the input-output 
firing rate relationship are varied from $p^{i a}$ and $r^{i a}$, for $r=$

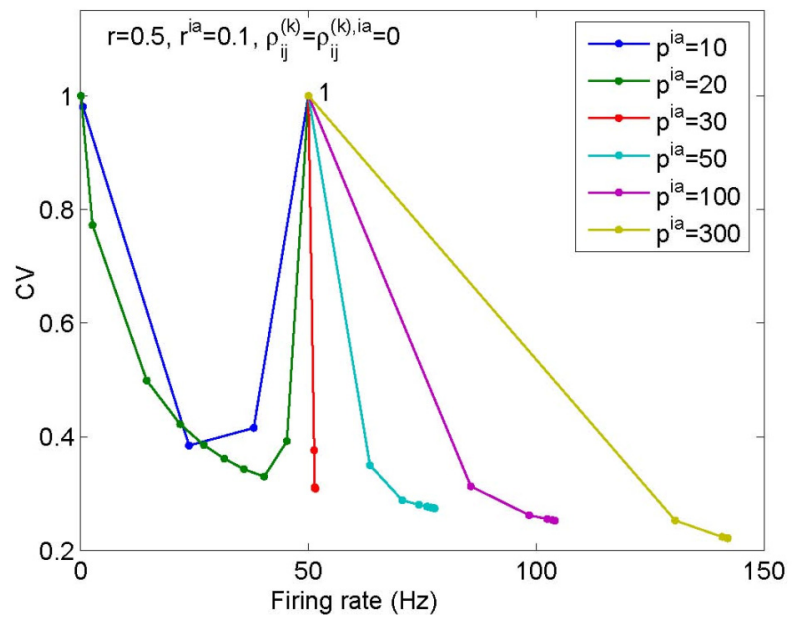

0.5 and $r=1.5$, respectively. As an analogous to [15],

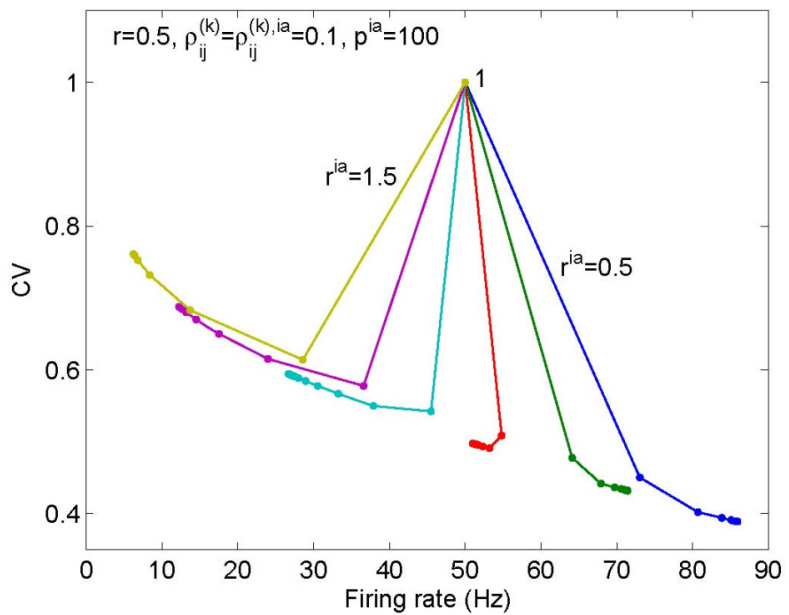

Fig. (2). Propagation of activity in a homogeneous MNN with context units, varied from numbers of intra-layer neurons $p^{i a}$ (left) and the ratio of intra- inputs $r^{i a}$ (right). Results were obtained for $r=0.5, p^{(k)}=p=100, \mu_{1}^{(1)}=50 \mathrm{~Hz}$.
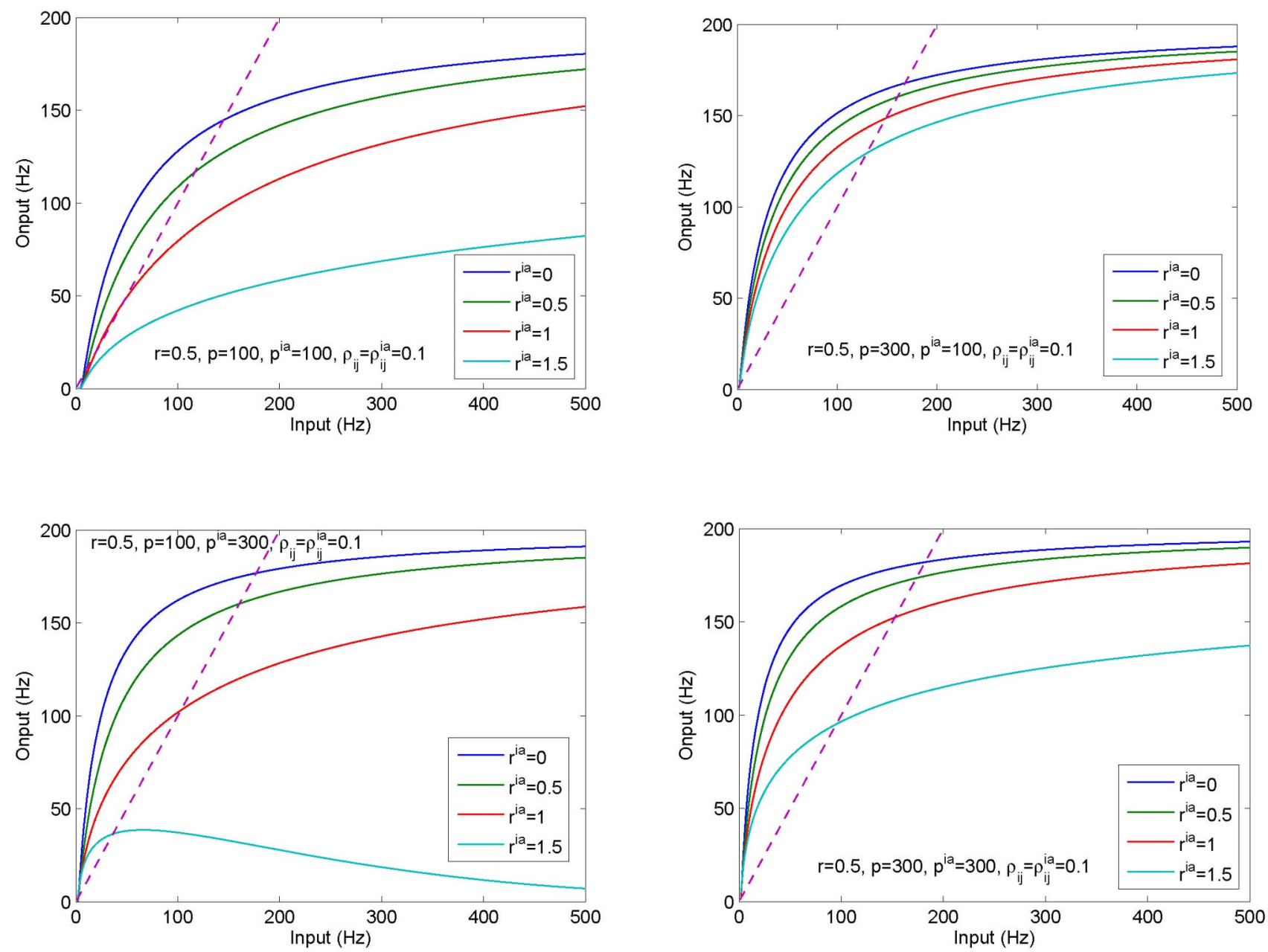
Fig. (3). The input-output firing rate relationship for a homogeneous MNN with context units, varied from numbers of intra-layer neurons $p^{i a}$ and the ratio of intra- inputs $r^{i a}$. Results were obtained for $r=0.5, \rho_{i j}=\rho_{i j}^{i a}=0.1$.
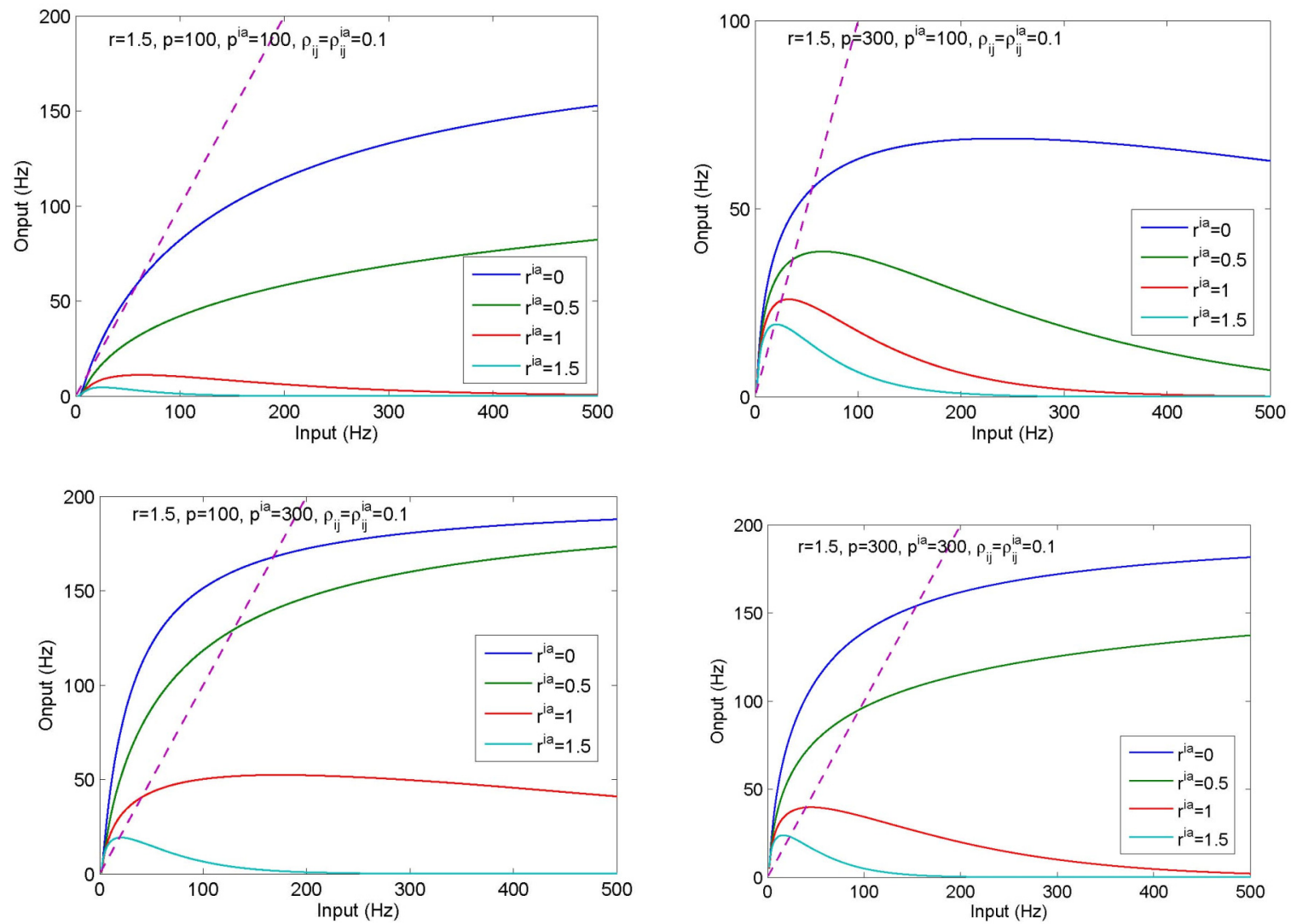

Fig. (4). The input-output firing rate relationship for a homogeneous MNN with context units, varied from numbers of intra-layer neurons $p^{i a}$ and the ratio of intra- inputs $r^{i a}$. Results were obtained for $r=1.5, \rho_{i j}=\rho_{i j}^{i a}=0.1$.
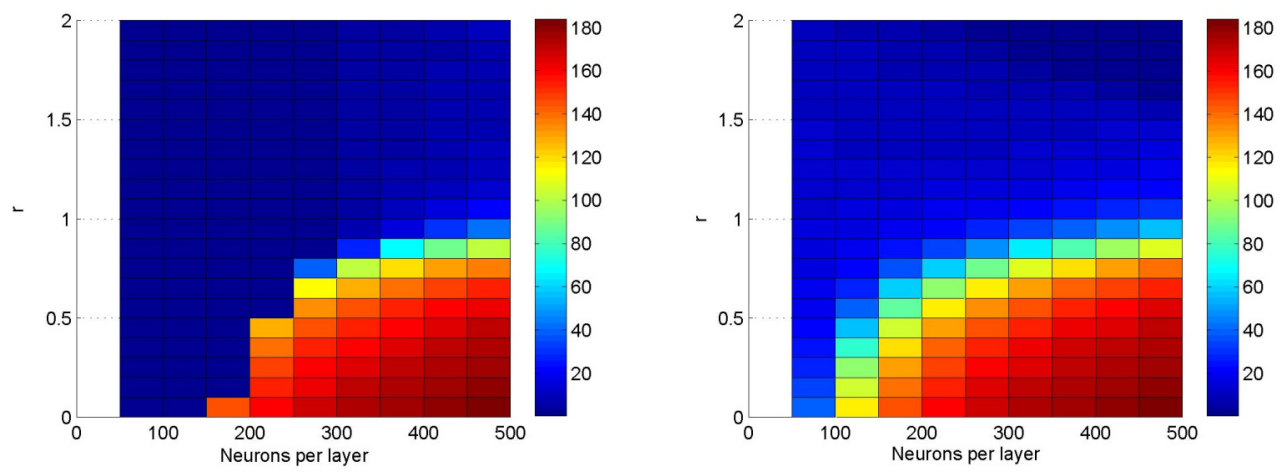

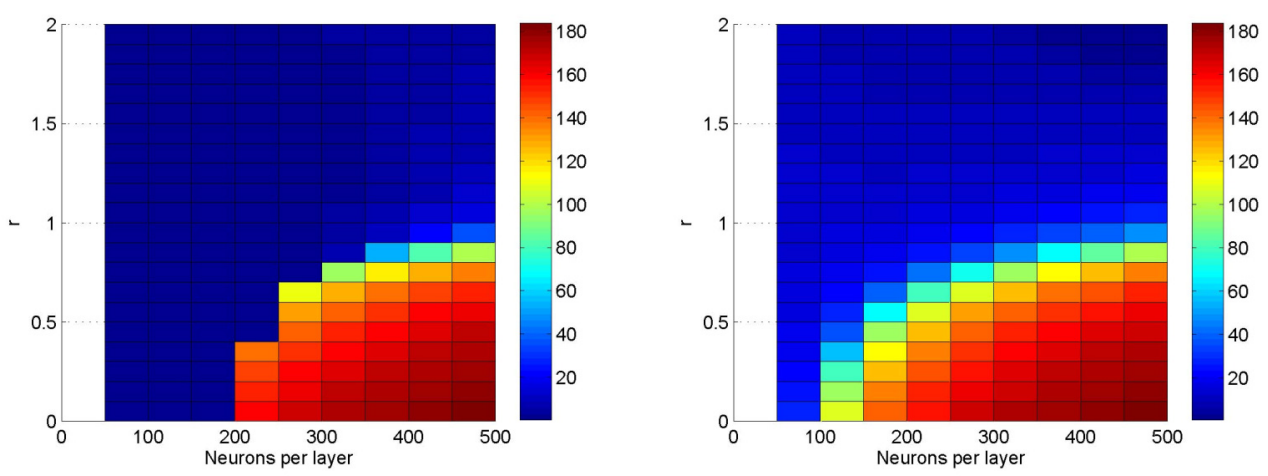

Fig. (5). Stable outputs for a homogeneous MNN with context units, varied from numbers of neurons $p$ and the ratio $r$. Results were obtained for $r^{i a}=0.8$ (top) and 1.5 (bottom), and $p^{i a}=100$ (left) and 300 (right), with $\rho_{i j}=\rho_{i j}^{i a}=0.1$.
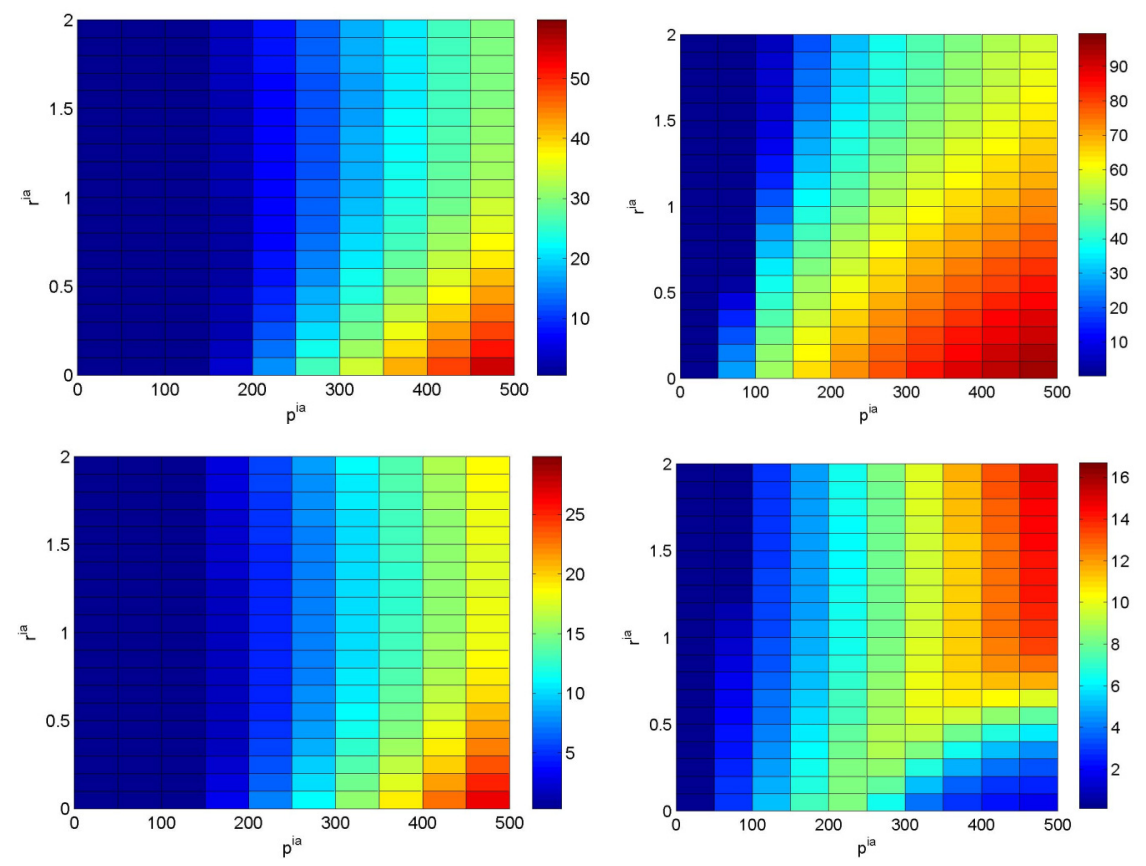

Fig. (6). Stable outputs for a homogeneous MNN with context units, varied from the number $p^{i a}$ and the ratio $r^{i a}$ of intra-layer neurons. Results were obtained for $r=0.8$ (top) and 1.5 (bottom), and $p=100$ (left) and 300 (right), with $\rho_{i j}=\rho_{i j}^{i a}=0.1$.
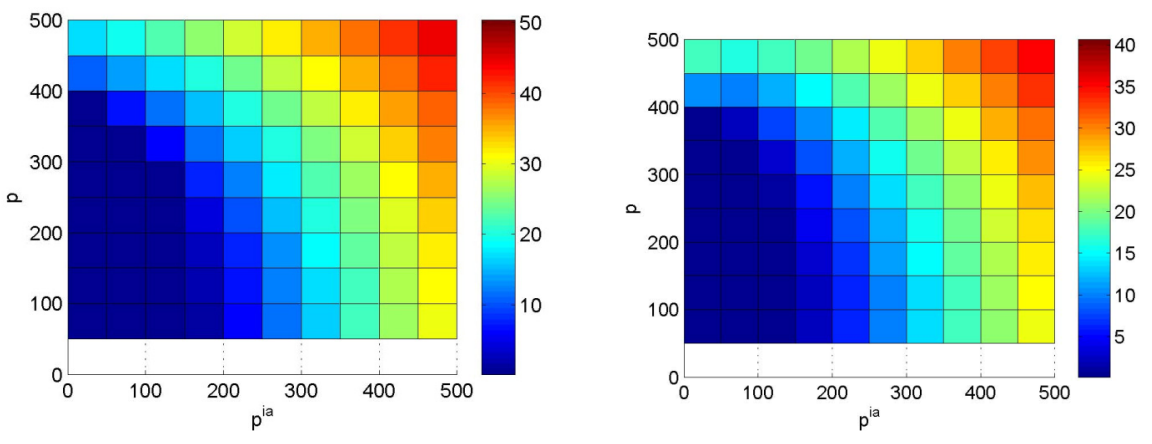

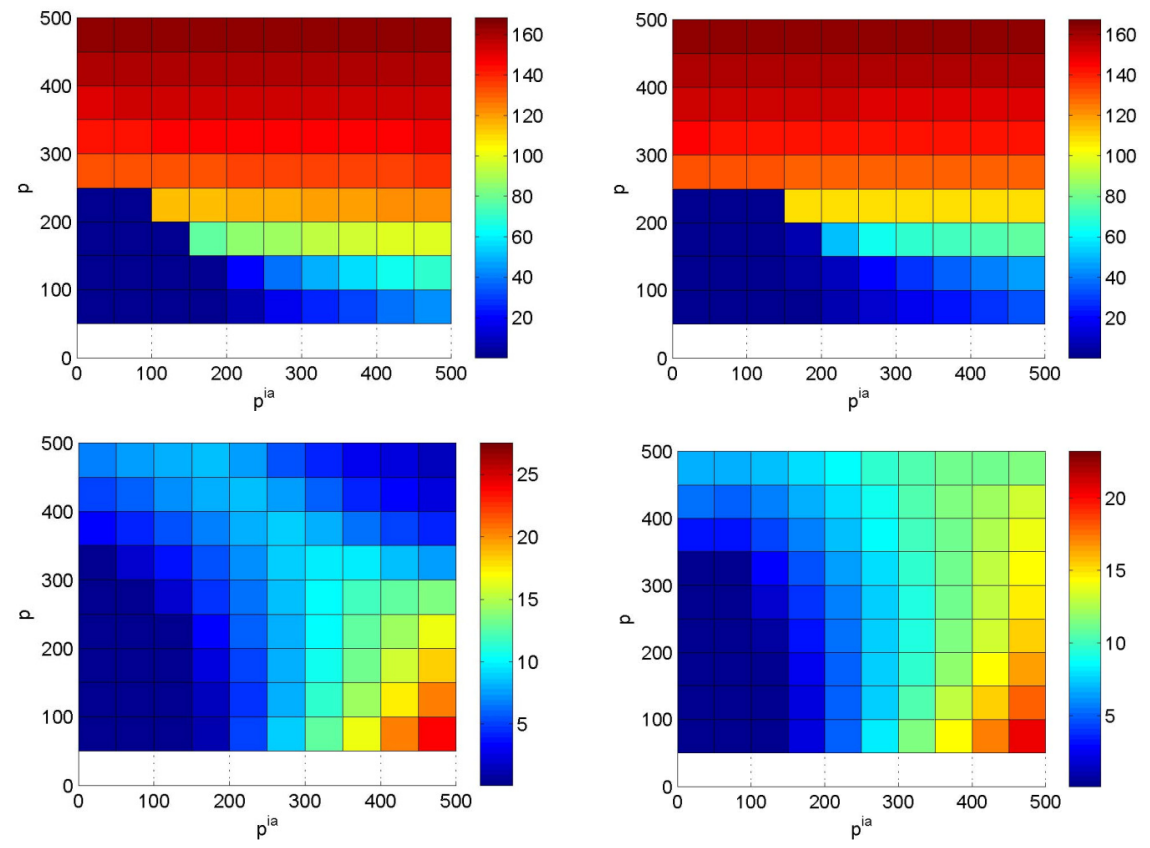

Fig. (7). Stable outputs for a homogeneous MNN with context units, varied from $p^{\text {ia }}$ of intra-layer and $p$ of inter-layer. Results were obtained for $r=1$ (top), $r=0.5$ (middle) and 1.5 (bottom), and $r^{i a}=0.5$ (left) and $r^{i a}=1.5$ (right), with $\rho_{i j}=\rho_{i j}^{i a}=0.1$.

roughly speaking, the stable solution appears at low firing rate for networks of increasing size (either $p$ or $p^{i a}$ size) with strong inhibition (either $r$ or $r^{i a}$ ). However the preferred appearance of stable solution at low frequency is not the increasing size of both inter- and intra- layer; see Fig. (4), for example, any ratio from intra- layer ( $r^{i a}$ varying from 0 to 1.5 ) appears a fix point at low firing rate when $p$ $=300, p^{i a}=100$ (top right), instead of $p=300, p^{i a}=300$ (bottom right).

Further we explore the stability of such network by showing the stable outputs varied from $(p, r)$ or $\left(p^{i a}, r^{i a}\right)$. Fig. (5) shows the stable outputs vs $r$ and $p$, with a fixed ratio from intra-layer inputs $r^{i a}=0.8$ (top) and $r^{i a}=1.5$ (bottom), ${ }_{i a}$ and with a number of neurons from intra-layer inputs $p^{i a}=100$ (left) and $p^{i a}=300$ (right). The initial inputs fix $5 \mathrm{~Hz}$ in the current and latter simulations. It shows that the ratio and number of neurons from intra- layer do not cause into a significant influence on the stability of networks, especially for $r>1$, the network will be either silent or firing at low frequency. As an analogue of Figs. $(5,6)$ shows the stable outputs vs $r^{i a}$ and $p^{i a}$, with $r$ $=0.8$ (top) and $r=1.5$ (bottom), and with $p=100$ (left) and $p=300$ (right). It is found that context unit (i.e. intralayer input unit) appears to have a sophisticated role in the stability of such network, depending on the ratio $r^{i a}$ and the size $p^{p a}$ of context unit, together with the ratio $r$ and the size $p$ of network itself, i.e. inter- layer. For example, for $r$ $=0.8$ (see top two panels), the stable output appears to increase with the increasing size $p^{i a}$ and the decreasing ratio $r^{i a}$ of context unit, which plays a general role.
Finally, we explore the stability of such network by showing the stable outputs varied from numbers of intralayer neurons $p^{i a}$ and of inter-layer $p$. Fig. (7) shows that the network with a small size of intra and inter-layer, will be either silent or firing at low frequency. Top panel shows that the stable output of such network seems to be symmetric between numbers of intra-layer and inter-layer when $r=1$. However there seen an obvious asymmetry for $r<1$ or $r>$ 1; see middle and bottom panels in Fig. (7). Middle panel shows that the network with a relatively large size of interlayer, for $r<1$, will be firing at relatively high rate, and that the size of intra-layer has negligible effect on the stable outputs such as $p>250$. Bottom panel presents unexpected results of $r>1$ that relatively high frequency is firing at the large size of intra-layer and small size inter-layer, rather than the large size of intra-layer and inter-layer. Especially for $p$ $>300$ and $p^{i a}>300$, the network is unexpectedly firing at low frequency when $r=1.5$ and $r^{i a}=0.5$; see the left of bottom panel. All those imply that the current network can lead to more sophisticated dynamics than MNN and the context unit plays very sophisticated role in dynamics of such network.

\section{DISCUSSION}

In the current paper we have focused on the dynamics of homogeneous feed-forward MNN with context units, akin to the dynamics of MNN. Due to a more biologically reasonable context units (i.e. intra- layer inputs), such network offers a significant advantage over the recent MNN in that it can change the dynamics of network by the adjustment of the parameters from context unit (and from network itself). It is obvious that the ratio between inhibitory 
and excitatory input, and the neurons number from both inter- and intra- layers are the parameters of such models (actually the weights and correlation coefficients are also in a heterogeneous network). As stated earlier, the context unit plays a very sophisticated role in the dynamics of such network. It can enhance or weaken the firing outputs by varying on the ratio $r^{i a}$ or the size $p^{i a}$ of context unit, based on the ratio $\mathrm{r}$ and the size $\mathrm{p}$ of network itself, i.e. inter- layer. It can lead to some unexpected dynamics and subsequently present some important gain in computational power over MNN.

\section{CURRENT \& FUTURE DEVELOPMENT}

In the future, a more sophisticated dynamics of heterogeneous network with context units will be explored. This might, for instance, shed light on how de-synchronization or synchronization is produced when we remove the constraint on the correlation coefficient between neurons.

\section{CONFLICT OF INTEREST}

The authors confirm that this article content has no conflict of interest.

\section{ACKNOWLEDGEMENTS}

The work was supported by National Natural Science Foundation of China (11171101), Hunan Provincial Natural Science Foundation (13JJ3114), Science and Technology Project Fund (2009GK3058), Scientific Research Fund of Education Department (10B073), and Scientific Research Fund of Hunan University of Atrs and Science (13ZD02).

\section{REFERENCES}

[1] B.W. Knight, "Dynamics of encoding in a population of neurons", J. Gen. Physiol., vol. 59, pp. 734-766, 1972.

[2] P. Rowcliffe, J. F. Feng, and H. Buxton, "Spiking perceptrons", IEEE Trans. Neural Netw., vol. 17, no. 3, pp. 803-807, 2006.

[3] H.C. Tuckwell, "Introduction to Theoretical Neurobiology", London: Cambridge University Press, 1988.

[4] J. Rouat R. Pichevar, S. Loiselle, Le T. T. Tai, Anh H. Hai, J.Lavoie, and J. Bergeron, "Spatio-temporal pattern recognition using a spiking neural network and processing thereof on a portable and/or distributed computer", U. S. Patent 8,346,692, Jan 01, 2013.

[5] M.S. Modha, "Neuromorphic and synaptronic spiking neural network with synaptic weights learned using simulation", U. S. Patent 8,515,885, Aug 20, 2013.

[6] R. Sarpeshkar, "Current-mode spike-based analog-to-digital conversion", U. S. Patent 6,262,678, Jul 17, 2001.

[7] R.E. Suri, "Applications of an algorithm that mimics cortical processing”, U. S. Patent 7,430,546, Sep 30, 2008.
[8] L.F. Abbott, and P. Dayan, "The Effect of Correlated Variability on the Accuracy of a Population Code", Neural Compute, vol. 11 pp. 91-101, 1999.

[9] J.F. Feng, and D. Brown, "Impact of correlated inputs on the output of the integrate-and-fire models", Neural. Comput., vol.12, pp. 671-692, Mar. 2000.

[10] F. Liu, J. F. Feng and W. Wang, "Impact of Poisson synaptic inputs with a changing rate on weak signal processing", Euro Phys. Lett., vol. 64, no. 1, pp.131-136, 2003.

[11] E. Salinas, and T. J. Sejnowski, "Correlated neuronal activity and the flow of neural information", Nat. Rev. Neurosci., vol. 2, pp. 539-550, 2001.

[12] M.N. Shadlen, and W. T. Newsome, "Noise, neural codes and cortical organization", Curr. Opin. Neurobiol., vol. 4, no. 4, pp. 569-579, 1994

[13] H. Sompolinsky, H. Yoon, K. J. Kang, and M. Shamir, "Population coding in neuronal systems with correlated noise", Phys. Rev. E, vol. 64, no. 5, p. 051904, 2001.

[14] E. Zohary, M. N. Shadlen, and W. T. Newsome, "Correlated neuronal discharge rate and its implications for psychophysical performance", Nature, vol. 370, pp.140-143, 1994.

[15] J.F. Feng, Y. C. Deng, and E. Rossoni, "Dynamics of moment neuronal networks", Phys. Rev. E., vol. 73, no. 4, 041906, 2006.

[16] X.Y. Xiang, and Y. C. Deng, "Training moment nenronal networks with intra- and inter-layer interactions", Inform. Int. Interdisciplinary $J$., vol. 15, no. 1, pp. 363-374, 2012.

[17] T.P. Trappenberg, "Fundamentals of Computational Neuroscience [M]". Oxford: Oxford University Press, 2002.

[18] F. Piekniewski, "Spiking neural network feedback apparatus and methods", WO Patent WO/2013/169805, Nov 14, 2013.

[19] X.Y. Xiang, Y. C. Deng, and X. Q. Yang, "Spike-rate perceptrons", Proceedings of Forth International Conference on Natual Computation, vol. 3, pp. 326-333, 2008.

[20] X.Y. Xiang, Y. C. Deng, and X. Q. Yang, "Second order spiking perceptrons", Soft Comput., vol.13, pp.1219-1230, 2009.

[21] X.Y. Xiang, Y. C. Deng, "The learning of moment neuronal networks", Neurocomputing, vol. 73, pp. 2597-2613, 2010.

[22] X.Y. Xiang, Y. C. Deng, "The learning of moment neuronal networks II", J. Comput. Inform. Syst., vol. 6, no. 11, pp. 3741$3749,2010$.

[23] X.Y. Xiang, Y. Chen, L. F. Liu, “An updated learning rule for moment neuronal networks", J. Inform. Comput. Sci., vol. 8, no. 13, pp. 2509-2516, 2011.

[24] A.S. Weigend, B. A. Huberman, and D. E. Rumelhart, "Predicting the future, a connectionist approach", Int. J. Neural Syst., vol. 3, pp. 193-209, 1990.

[25] D.A. Winkler, and F. R. Burden, "Bayesian neural nets for modeling in drug discovery", Drug Discov. Today, BIOSILICO, vol. 2, no. 3, pp.104-111, 2004.

[26] K. Arai, "Learning method for recursive neutral network and recording medium where learning program for recursive neural network is recorded", J. P. Patent 2,000,200,256, Jul 18, 2000.

[27] W. Gerstner, and W. Kistler, "Spiking Neuron Models", Cambridge: Cambridge University Press, 2003.

[28] J.F. Feng, "Computational Neuroscience-A Comprehensive Approach", Chapman-Hall/CRC Press, 2003.

[29] V. Livak, H. Sompolinsky, I. Segev, and M. Abeles, "On the transmission of rate code in long feedforward networks with excitatory inhibitory balance", J. Neurosci., vol. 23, pp. 3006-3015, 2003. 